\title{
Endogenous testosterone is associated with increased striatal response to audience effects during prosocial choices:
}

\author{
Yansong Li $\mathrm{Li}^{\mathrm{a}, \mathrm{b \#}}$, Elise Météreau ${ }^{\mathrm{c}, \mathrm{d}}$, Ignacio Obeso ${ }^{\mathrm{c}, \mathrm{d}, \mathrm{e}}$, Luigi Butera ${ }^{\dagger}$, Marie Claire Villeval $^{\mathrm{g}}$ and \\ Jean-Claude Dreherc, d\# \\ a Reward, Competition and Social Neuroscience Lab, Department of Psychology, School of \\ Social and Behavioral Sciences, Nanjing University, Nanjing 210023, China \\ b Institute for Brain Sciences, Nanjing University, Nanjing 210023, China \\ ${ }^{\mathrm{c}}$ Neuroeconomics, Reward and decision making' group, Cognitive Neuroscience Center, CNRS \\ UMR 5229, Bron, France \\ dUniversité Claude Bernard Lyon 1, Lyon, France \\ e HM Hospitales - Centro Integral en Neurociencias HM CINAC, Móstoles, Madrid, Spain \\ ${ }^{\dagger}$ Copenhagen Business School, Denmark \\ g Univ Lyon, CNRS, GATE (UMR 5824), Ecully 69130, France
}

Running Title: Testosterone and striatal response to audience effects

\section{"Correspondence:}

Yansong Li, Ph.D

Reward, Competition and Social Neuroscience Lab

Department of Psychology \& Institute for Brain Sciences

Nanjing University

Nanjing, China

Telephone: 0086-025-89680958

Fax: 0086-025-89680950

Email: yansongli@nju.edu.cn

Jean-Claude Dreher, Ph.D

Neuroeconomics Lab,

Institut des Sciences Cognitives Marc Jeannerod, CNRS

67 Bd Pinel, 69675 Lyon

France

Tel: +33 (0)437911238

Fax:+33 (0)437911210

Email: dreher@isc.cnrs.fr 
44 The role of testosterone on cognitive functions in humans remains controversial. One recent

45 hypothesis suggests that this steroid hormone advances social status. As being observed by 46 others is known to modulate a range of behaviors because of image concerns, we

47 hypothesized that such an audience effect might be an important component of status 48 seeking that is under the control of testosterone. Thus, we investigated to which extent 49 testosterone levels are associated with the effect of being observed during prosocial choices 50 and the neural mechanisms underlying this effect. We enrolled twenty-four male participants, 51 aged $22.47 \pm 2.62$ years, in an $\mathrm{fMRI}$ experiment to examine the relationship between 52 testosterone levels and brain activity engaged in deciding whether to accept or reject 53 monetary transfers to two types of organizations (a positively evaluated organization and a 54 negatively evaluated organization) in presence or absence of an audience. When comparing 55 the public to the private condition, the rate of acceptance increased for the positively 56 evaluated organization, while the rate of rejection increased for the negatively evaluated one. 57 Higher testosterone levels were linked to greater activation in the striatum in the public 58 compared to the private condition, regardless of the organization type. These results

59 indicate a relationship between testosterone levels and striatal activity induced by the 60 audience effect. These findings provide new insights on the role of testosterone in human 61 social behavior.

63 Keywords: Testosterone; Audience effect; Striatum; Social image; Charitable giving 


\section{Introduction}

The steroid hormone testosterone has long been known to regulate the development of

68 physical masculinization (Renfree et al., 2002). Apart from its role in the body, there has been

69 growing interest in understanding testosterone-behavior relationships over the past decades

70 (Geniole and Carré, 2018; Hines, 2017). One traditional view on testosterone functions is that

71 it drives certain forms of aggression in both humans (Coccaro et al., 2007; Dabbs and

72 Hargrove, 1997; Räsänen et al., 1999) and non-human primates (Bouissou, 1983;

73 Giammanco et al., 2005). However, this traditional view of the role of testosterone in driving

74 aggression has been revisited in more recent theories and experiments (Archer, 2006;

75 Nadler et al., 2019). Recent studies emphasized its relation to status-enhancing behavior in

76 the form of prosocial or antisocial behavior, depending on the social contexts (Booth et al.,

77 2006; Dreher et al., 2016; Eisenegger et al., 2011; Mazur and Booth, 1998). For example,

78 higher levels of testosterone in both men and women have been associated with enhanced

79 social status (Rowe et al., 2004; Sellers, 2006) or increased spatial cognitive skills when

80 status is at play (Newman et al., 2005). Other behavioral results in men and women have also

81 emphasized the relationship between testosterone levels and social cooperation (Casto and

82 Edwards, 2016; Sanchez-Pages and Turiegano, 2010) or the choice of an interaction

83 strategy (domination vs. submission) in a social context (Inoue et al., 2017; van Honk et al.,

84 2014). In addition to these correlational evidence, recent behavioral studies tested to what

85 extent testosterone administration plays a causal role during social interactions. A single

86 dose of testosterone in women decreased trust but increased generosity in non-competitive

87 settings (Boksem et al., 2013), led to fair bargaining behavior (Eisenegger et al., 2010) and

88 motivated for reputable-status seeking, even when the resulting behaviors were

89 economically disadvantageous (van Honk et al., 2016). Similarly, these findings have been

90 extended to men. For example, exogenous testosterone administration in men has been 
91 shown to increase not only the altruistic punishment of unfair offers, but also prosocial

92 behavior (positive reciprocity) in response to generous offers in a modified ultimatum game

93 (Dreher et al., 2016), social cooperation (van Honk et al., 2012), preferences for high-status

94 goods (Nave et al., 2018) and status-seeking motivation with unstable low social status

95 (Losecaat Vermeer et al., 2020).

96 However, a key element of social interactions in real-world settings is whether other

97 individuals can observe both the decisions made by the decision maker and their consequences, which is in fact a neglected aspect of the aforementioned studies. Decisions under observability can indeed be influenced by individuals' image concerns. In these 100 settings, individuals may focus on matching their in-group social values rather than raising 101 social status (Everett et al., 2015). Previous studies have found that individuals' behavior 102 can be influenced by the mere presence of others (Hamilton and Lind, 2016), suggesting 103 that the presence of an audience may be one of the dominant factors driving several social 104 enhancing behaviors (Bradley et al., 2018). Audience as a modulator of behavior has been 105 found in a diversity of species, including humans and nonhuman primates (Chib et al., 2018; 106 Sekiguchi and Hata, 2018). Given that the mere presence of an audience can promote 107 status-seeking behavior in our social life and testosterone has been shown to play an 108 important role in status-relevant behavior, understanding the extent to which testosterone 109 levels can be related to audience during prosocial decisions would greatly advance our 110 understanding of testosterone-behavior relationships. In particular, since testosterone is 111 involved in status-relevant behavior, one may expect that an audience should enhance its 112 relation with norm-compliant prosocial behavior. Moreover, identifying the underlying neural 113 mechanisms of the association between testosterone levels and audience in prosocial 114 behavior would provide important insights not only into the prosocial role of testosterone in 115 the context of social interactions, but also into the mechanisms underlying the 
116 testosterone-status relationship. This matters particularly since testosterone has been shown

117 to be disrupted in psychiatric disorders (Li et al., 2020). In particular, children who have been

118 exposed to high concentrations of testosterone as a fetus would be more likely to exhibit

119 autistic traits (Mullard, 2009). Although previous research investigated the effect of audience

120 on prosocial behavior in autism, the relationship with testosterone remains to be investigated

121 (Izuma et al., 2011). Prior neuroimaging evidence pinpoints a brain network essential for

122 conducting prosocial decisions. This includes the striatum, anterior cingulate cortex (ACC),

123 ventromedial prefrontal cortex (vmPFC) and temporo-parietal junction (TPJ). This network is

124 recruited when expecting social rewards as well as when weighing monetary costs against

125 compliance with one's moral values, or when helping choices are made (Cutler and

126 Campbell-Meiklejohn, 2019; Qu et al., 2019). Yet, how the aforementioned network is

127 regulated by sex hormone is unknown, although an increasing effort has been devoted to

128 exploring how other hormones such as estrogen and oxytocin modulate prosocial behavior

129 (Kemp and Guastella, 2010; Zethraeus et al., 2009). Here, we explored the relationship

130 between endogenous testosterone levels and the neural mechanisms underlying prosocial

131 behavior in reaction to the presence or absence of an audience. To address this question,

132 we used the behavioral data from a donation experiment published by Qu et al. (2019). In

133 this experiment, participants had to decide whether to accept or reject monetary transfers to

134 two organizations (one positively evaluated, and the other negatively evaluated). Prosocial

135 behavior was characterized by two types of decisions: accepting a monetary transfer to a

136 positively evaluated organization at a personal cost, or foregoing personal monetary gains to

137 reject a transfer to an organization that they evaluated negatively. These decisions were

138 made in private or in public, depending on the trials. Decisions while being observed

139 required weighing the costs and benefits of accepting vs. rejecting the donation, plus the

140 expected (positive or negative) image sent to the observer. Such reasoning requests the 
141 conversion of social and monetary rewards into a common currency for comparisons to be 142 made (Sescousse et al., 2015). In such settings, participants thus faced a moral dilemma:

143 either serving a good cause but at a personal monetary cost, or making money but betraying 144 ones' moral values. This design allows us to investigate whether testosterone is involved in 145 guiding prosocial vs. selfish decisions induced by the presence of an audience when 146 participants face a moral dilemma. Because weighing monetary costs against compliance 147 with one's moral values (Qu et al., 2019) and perceiving one's good reputation (Izuma et al., 148 2008, 2010) have been reported to result in striatal activity, we hypothesize a positive 149 correlation between testosterone levels and striatal activation while making prosocial 150 decisions in reaction to the presence of an audience. 


\section{Material and methods}

\subsection{Participants}

We summarize in this section the experimental design, all the details being developed in Qu et al. (2019). Twenty-four healthy male participants, aged $22.47 \pm 2.62$ years, with no history of neurological or psychiatric illness participated in the fMRI experiment. Three participants were discarded from the analysis because of failure to collect testosterone data. All participants were right-handed, as assessed by the Edinburgh Handedness Inventory (Oldfield, 1971), and presented no symptoms of depression, as assessed by the 13-item version of the Beck Depression Inventory (Beck and Beck, 1972). Informed consent was obtained from every participant. The study was approved by the local ethics committee (CPP Centre Léon Bérard).

\subsection{Pre-testing}

As described in our previous study (Qu et al., 2019), a behavioral pilot study involving 48 healthy volunteers was performed at GATE-Lab, Lyon, to help with designing stimuli and task procedures. To guide the selection of the organizations, we asked them to complete a questionnaire after the presentation of brief descriptions and logo images of 14 organizations. Organizations with positive or negative valence were presented. For each one, participants had to rate their feelings towards them on a scale from -10 to 10 . The organizations were presented in the questionnaire in a random order across participants. Based on this pilot study, we chose for the $\mathrm{fMRl}$ experiment the two organizations that received the worst $($ mean $=-5.73, S D=3.68)$ and the best $($ mean $=8.40, S D=2.04)$ ratings. They were a negatively evaluated organization (NEG ORG) ('Groupe d'Action Royaliste', an organization that aims at promoting the restoration of monarchy in France) and a positively evaluated charity (POS ORG) ('Resto du coeur', a charity providing food to poor people). Because the policy does not allow us to publish trademarked names, we have 
changed the real names of these two organizations. GAR represents the NEG ORG and RES (a symbol of heart) represents the POS ORG (a charity providing food to poor people) (Fig 1).

\subsection{Experimental Task}

Our previous study (Qu et al., 2019) described that "we used a $2 \times 2$ within-participant design, in which participants decided whether to accept or reject monetary transfers to the two organizations. Depending on the blocks of decisions, the offers of transfer is concerned with either the POS ORG or the NEG ORG. Decisions were made either in presence or absence of observers ("public" vs. "private" conditions) (Fig 1). At the beginning of the experiment, participants received an initial endowment of 14 Euros. During the experiment, they were faced with successive offers involving a variable monetary payoff for themselves and a variable payoff for the organization. When making decisions regarding the POS ORG, participants had to decide whether to accept or reject monetary transfers to the organization at a variable monetary cost to themselves, deducted from their initial endowment. When making decisions regarding the NEG ORG, they had to decide whether to accept or reject monetary transfers to the organization in exchange for a personal monetary payoff added to their initial endowment. In the latter case, the only way for a participant to earn money was to accept a donation to the NEG ORG, whereas in the former treatment, any donation to the POS ORG involved a monetary loss for the participant. One crucial aspect is that in both treatments, each organization would receive a donation; however, in one case such a donation entails a moral cost for the individual (allowing the experimenter to send money to the NEG ORG in order to earn money for oneself may violate one's moral values), while in the other case, the donation to the organization generates a moral benefit for the individual (altruistically foregoing a personal gain to benefit the POS ORG may comply with one's moral values). Because we systematically varied the monetary cost of a moral decision, we 
203 were able to identify the price elasticity of demand for moral actions. Intuitively, if participants

204 did not perceive some actions as immoral, they would display no elasticity to the moral cost

205 of choosing the self-serving action. The monetary stakes for the organizations and for the 206 participants varied independently across trials. In each trial, the organization's potential 207 gains ranged from 4 to 32 Euros, in increments of 4 Euros. Participants' potential payoffs (in 208 the case of the NEG ORG) or costs (in the case of the POS ORG) varied from 1 to 8 Euros, 209 in increments of 1 Euro. Each participant was therefore exposed to 64 different dilemmas.

210 Only one public decision and one private decision among all the trials were randomly 211 selected for payment at the end of the experiment. If the participant accepted the offer in the 212 randomly selected trial, the amount of the accepted transfer was sent to the organization 213 (the mean of the two amounts was used if the two trials concerned the same organization), 214 and the participant's endowment was increased or decreased based on his decision. If the 215 same organization happened to be randomly selected twice, then the organization received 216 the average transfer and the participant's endowment was adjusted based on the average of 217 the two decisions. If the participant rejected the offer in the randomly selected trials, nothing 218 was sent to the organization, and the participant's initial endowment was not modified.

219 The presence or absence of an observer (public versus private conditions) was 220 displayed on the screen in the following way. In private trials, a yellow frame surrounded the 221 offer, and a picture of a padlock was displayed at the top of the screen reminding 222 participants about the privacy of their decisions. In the public condition, a cyan frame 223 surrounded the offer, and a picture of the eyes of an observer was displayed above, 224 reminding participants that an independent observer would see their decisions. Indeed, cues 225 of being watched exert an influence on participants' behavior (Bateson et al., 2006). To 226 further stress the visibility of their choices in the public trials, participants knew that an 227 observer in the control room, to whom they were introduced prior to the experiment, would 
228 see the participant's screen and therefore observe their public trials decisions; in the public 229 trials, the chosen alternative was highlighted for $1.5 \mathrm{~s}$ on the screen by expanding the font, 230 while the other option disappeared. In the private condition, no changes were made on the 231 screen after the response, assuring participants that nobody would be able to see their 232 choices from the scanner control room. Finally, at the end of the experiment, participants 233 had to declare in front of a video camera which decision they made in the randomly selected 234 trial for the public condition. Participants were told that decisions in the private condition 235 were recorded anonymously, guaranteeing that none of the experimenters could link a 236 participant's identity with his decisions. A person not affiliated with the experiment and 237 unaware of its content paid all participants. All the participants reported believing in the 238 manipulation.

239 For each possible combination of individual and organization payoffs, and for both 240 organizations, participants made two decisions, one in private and one in public. Participants 241 therefore made a total of 256 decisions, 128 related to the NEG ORG and 128 related to the 242 POS ORG. Each trial began with the presentation of an offer, which could either be accepted 243 or rejected by pressing the left or right button on a response pad. A fixation cross was 244 displayed during a random time interval (jitters), drawn from a uniform distribution between

2452.5 and 6.5s. Participants were encouraged to make their decision within $3 \mathrm{~s}$. After this delay, 246 a message was displayed on the screen to remind them to respond.

247 The scanning session was divided into 4 runs of 64 trials. The first two runs concerned 248 one organization and the last two concerned the other organization. Within the first run of 249 each organization, the first half of the trials was either public or private, with the opposite for 250 the subsequent run. The order of the private/public conditions in the second run mirrored the 251 order of these conditions in the first run. The order of presentation of the organizations and 252 of public/ private conditions was balanced across participants. Thirty-two dilemmas from the 
25364 possible combinations were presented in each run and each private/public condition. To

254 guarantee that the two pairs of runs of each organization were balanced with respect to the 255 payoffs for the individual and the organization, we assigned to one run the set of dilemmas 256 composed by the participant's odd potential payoffs and the $4,12,20$, and 28 potential 257 amounts for the organization, while the other run was assigned the 32 remaining dilemmas 258 of the matrix. Within this criterion, the order of the 32 dilemmas was randomized.

259 Visual stimuli were back-projected on a screen located at the head of the scanner bed 260 and presented to the participants through an adjustable mirror located above their head. The 261 presentation of the stimuli was controlled by Presentation () software (Neurobehavioral 262 Systems), which also recorded trigger pulses from the scanner signaling the beginning of 263 each volume acquisition."

\section{$264 \quad 2.4$ Procedures}

265 During a first interview (the pilot pre-testing), participants were asked to rate their 266 feelings toward each of 14 organizations on a scale ranging from -10 to 10 . Based on this 267 pilot study, we chose for the fMRI experiment the two organizations that received the worst 268 and the best ratings. For the fMRI experiment, we selected only participants who rated the 269 POS ORG with a score greater than 0 and the NEG ORG with a negative score. The day of 270 the experiment, participants first received instructions about the experiment.

271 After receiving the instructions, participants did a few free practice trials of all conditions 272 in the control room of the fMRI and were allowed to ask questions. After the practice session, 273 participants were asked to read a description of the two organizations. Before entering the 274 fMRI room, they met with the independent observer. After scanning, the participants were 275 debriefed. Participants filled a post-experimental questionnaire asking whether they truly 276 perceived the different trials as independent, whether they believed in the difference 
between private and public conditions, and whether they thought that the presence of the observer had influenced their decisions.

\subsection{Testosterone Measurements}

In order to minimize the effect of circadian hormone rhythms, all sessions were 281 conducted between 1:45 PM and 3:45 PM. Prior to and after the scanning session, blood 282 samples were obtained to detect the levels of plasma testosterone for each participant. 283 Plasma total testosterone was used for the assay and was measured by a solid-phase, 284 competitive chemiluminescent enzyme immunoassay, IMMULITE 2000 (Diagnostic 285 Products Corporation, Los Angeles, CA). Intra- and inter-assay coefficients of variation were $2867.2 \%$ and $8.2 \%$, respectively. Such an assay had an analytical sensitivity of $0.5 \mathrm{nmol} / \mathrm{L}$. 287 Corrections for incomplete recovery were made using $3 \mathrm{H}$-labeled internal standards 288 (Déchaud et al., 1981; Rinaldi et al., 2001; Sabot et al., 1985). Free testosterone would be 289 more interesting to investigate, but we did not record sex-hormone binding globulin (SHBG) 290 allowing to compute free testosterone values. In spite of this, the measurement of total 291 testosterone has still been argued to be effective in exploring the potential link between 292 testosterone levels and neuropsychological functions in humans (Hua et al., 2016). In order 293 to control for other variables affecting testosterone levels, participants were asked to 294 practice little physical exercise during the appointment day and to refrain from any 295 caffeine-containing food or drinks and cigarettes from at least one hour before the 296 experiment started.

\section{$297 \quad 2.6$ Behavioral Analysis}

298 We characterized accepted trials in the POS ORG and rejected trials in the NEG ORG 299 as "prosocial selection", as these two options permit to a positively evaluated charity to earn 300 money or avoid that a negatively evaluated organization receives money at a personal direct 
301 or indirect cost to the participants (either through a reduction of the initial endowment or

302 through foregoing a potential gain). By contrast, the rejected trials in the POS ORG and the 303 accepted trials in the NEG ORG were both characterized as "selfish selection" because 304 these options increased or preserved the initial endowment. Our previous study (Qu et al., 305 2019) has reported in detail the relationships between the parameters of the tasks and 306 participants' decisions, identified by using random-effects logistic models for each 307 organization. Therefore, here we only report a brief and updated analysis of the main 308 findings after having excluded the three participants from our previous study for whom we 309 failed collecting hormones. A repeated-measures ANOVA on prosocial choices was 310 conducted, with audience condition (public vs. private) and organization type (POS vs. NEG 311 ORG) as within-participants factors. This is followed by Wilcoxon signed-rank tests for 312 post-hoc testing.

\section{$313 \quad 2.7$ fMRI Data Acquisition}

314 The details of the fMRI acquisition and analysis have been reported in Qu et al. (2019). 315 fMRI data was acquired on a 1.5 Tesla Siemens MRI scanner. The scanning was divided 316 into 4 sessions. Blood-oxygenation-level-dependent (BOLD) signal was measured with 317 gradient echo T2* $^{*}$ weighted echo-planar images (EPIs). Twenty-six interleaved slices 318 parallel to the AC-PC line were acquired per volume (matrix $64^{\star} 64$, voxel size $=3.4^{\star} 3.4^{\star} 4$ $319 \mathrm{~mm}, \mathrm{TR}=2500 \mathrm{~ms}, \mathrm{TE}=60 \mathrm{~ms})$. We used a manual shimming within a rectangular region 320 including the orbitofrontal cortex and the basal ganglia to improve the local field 321 homogeneity. A high-resolution T1-weighted structural scan was subsequently acquired for 322 each participant (matrix $256 \times 256 \times 176$; voxel size $=1 \times 1 \times 1 \mathrm{~mm}$; TR = 1,970 $\mathrm{ms}$; TE = $3233.93 \mathrm{~ms} ;$ flip angle = 15).

\section{$324 \quad 2.8$ fMRI Pre-processing}


Data were pre-processed and analyzed using the SPM8 software package (Wellcome

326 Department of Imaging Neuroscience, London) implemented in Matlab 7.10 (Mathworks,

327 Natick, MA). The first four functional volumes of each session were removed to allow the 328 BOLD signal to reach a steady state. The remaining images were slice-timing corrected, 329 spatially realigned and unwarped to correct for motion artifacts. Unwarping was performed 330 based on phase maps calculated using the Fieldmap SPM toolbox. Then in order to 331 suppress the residual fluctuations due to interpolation errors from large motions, we used 332 the motion adjustment algorithm provided in the ArtRepair toolbox (Mazaika et al., 2009) 333 after a smoothing with a $4 \mathrm{~mm}$ full width at half maximum (FWHM) Gaussian kernel. This 334 method is an alternative to adding motion regressors to the design matrix. The scan artifacts 335 were then detected and repaired using both global intensity and scan-to-scan movement with the Artifact Repair algorithm from the ArtRepair SPM toolbox.

For each participant, the structural image was co-registered to the mean functional 338 image, segmented into white and gray matter, and the gray matter was normalized to a 339 standard gray matter template distributed by SPM8. The transformation parameters 340 estimated in this step were applied to all functional images. Functional images were then 341 spatially smoothed with a $7 \mathrm{~mm}$ FWHM Gaussian kernel.

\section{$342 \quad 2.9$ fMRI Data Analysis}

343 As described in our previous study (Qu et al., 2019), at the single-participant level, 344 statistical analyses were performed using a GLM in which all regressors were modeled as 345 delta functions and convolved with a canonical hemodynamic response function (HRF). We 346 applied a high-pass filter with a cut-off of $128 \mathrm{~s}$ to the time series to remove low-frequency 347 noise and baseline drifts, and we used an $\operatorname{AR}(1)$ model plus white noise to correct for 348 temporal autocorrelation. Estimations were done in an explicit grey matter mask based on 349 the tissue probability map provided by SPM. 
Since the current study aims at exploring the relationships between testosterone levels

351 and brain activity involved in the audience effect, we need to describe the analysis of 352 audience effects based on our previous study (Qu et al., 2019). Specifically, we focused on a 353 number of brain regions, such as those associated with making prosocial choices in the 354 charity condition and those engaged with an audience effect, regardless of organization 355 types or choices. We attempted to build a model including 8 regressors of interest at the time 356 of "offer onset" in separate conditions 2 (accepted trials vs. rejected trials) $\times 2$ (private vs. 357 public) $\times 2$ (POS vs. NEG ORG). We included the size of the potential gain for the 358 organization and the size of the potential gain or loss for the participant with two orthogonal 359 parametric regressors. Because little is known about the brain networks engaged when 360 being observed (i.e., in the public condition) compared to when making decisions in private, 361 regardless of the choice made, we performed two contrasts to test for the main effects of 362 audience and privacy: public > private, and private > public, regardless of the organization 363 types and participants' choices. Given our specific a priori region of interest, we used small 364 volume correction (SVC) with a threshold of $\mathrm{P}<0.05$ (FWE corrected) based on our a priori 365 region of interest. The SVC was performed using a sphere with $10 \mathrm{~mm}$ radius centering 366 around the coordinate of peak voxel in the left and right putamen (left: -16, 14, -10; right: 12, 367 10, -4) derived from a previous studies on audience effect (Izuma et al., 2010) and in the left 368 and right caudate nucleus $(x, y, z=-17,6,13$ and $x, y, z=18,6,9)$ derived from a previous 369 study where charitable donation was investigated (Moll et al., 2006). Please note that these 370 original coordinates in the Talairach space were transformed into the corresponding 371 coordinates in MNI space using GingerALE 2.3. Given that we ran four SVC tests restricted 372 to a single region, we have used a Bonferroni-corrected threshold of $0.05 / 4=0.013$, 373 accounting for the number of SVC tests. 
374 For the correlational analysis between testosterone levels and striatal activity induced by 375 the public vs. private contrast for both organizations, we employed the averaged 376 testosterone levels between those measured prior to and after the scanning session in a 377 simple regression analysis. To illustrate the correlation between testosterone levels and the 378 patterns of activation, percentage signal changes were extracted in the functional ROls of 379 interest (left caudate and left putamen) using the MarsBar toolbox 380 (http://marsbar.sourceforge.net). 
382

383

384

385

\section{Results}

\subsection{Audience effects}

Our ANOVA analysis showed that there was a significant main effect of organization type on prosocial choices $(F(1,20)=7.50, p<0.05)$, whereas there was not a significant main effect of audience condition on prosocial choices $(F(1,20)=0.01, p>0.05)$. Moreover, there was a significant interaction between them on prosocial choices $(F(1,20)=8.79, p<0.01)$. A Wilcoxon signed-rank test showed in the POS ORG, participants accepted significantly more offers on average in the public $(70 \%)$ as compared to the private condition $(66 \%$; Wilcoxon $|Z|=2.81, p<0.01, r=0.43)$. In contrast, in the NEG ORG, participants accepted significantly less offers on average in the public $(43 \%)$ relative to the private condition (47\%; Wilcoxon $|Z|=2.30, p<0.05, r=0.35)($ Fig 2A). This was further confirmed by color-coded heatmaps of the probability of accepted donations for transfers to the POS ORG and the NEG ORG, respectively (Fig 2B). These color-coded heatmaps clearly demonstrated that participants were more willing to accept to donate to the POS ORG in public than in private condition and were less willing to accept to donate to the NEG ORG in public than in private condition.

\subsection{The link between testosterone, behavior and striatum}

We first analyzed the main effect of acceptance in the public compared to the private condition, independently of the organization type. Striatal activity significantly increased in public compared to private decisions (MNI [x y z] [-12 2 -2], $T=3.66, p(S V C)<0.05$, FWE) (Table 1). In addition, regions such as the anterior cingulate cortex (ACC) (MNI[x y z] [0 23 34], $\mathrm{T}=5.65)$, temporal parietal junction (TPJ) (MNI[x y z] [48 -25 25], $\mathrm{T}=4.34$ ) were also active in public vs private decisions (Table 1). By contrast, in private vs public decisions, a different brain network was found with only the occipital gyrus (MNI[x y z] [30 -82 -20], T= 4.45) being significantly engaged (Table 1). Given that our a priori hypothesis predicts a 
407 positive correlation between testosterone levels and striatal signal during prosocial decisions 408 in presence of an audience, we performed a correlation analysis between testosterone 409 levels and BOLD responses for prosocial decisions made in public vs in private for both 410 types of organization. As predicted, our results revealed a positive relationship between 411 testosterone levels and striatal activity induced by prosocial decisions for public $>$ private 412 condition (putamen: MNI[x y z] [-21 5 -11], T=6.77, $p(S V C)<0.05$, FWE; caudate nucleus: 413 MNI[x y z] [-15 2 13], T = 5.07, $p($ SVC) < 0.05, FWE) (Fig 3; Table 2). The striatum, involved 414 in prosocial behavior in public, showed a correlation with endogenous testosterone levels.

415 By contrast, when looking at prosocial decisions made in private $>$ in public for both types of 416 organization, we found no supra-threshold activations in the social image-related brain 417 network correlating with testosterone levels (Table 2). Meanwhile, to exclude potential 418 confounding effects caused by the salience of the public context per se, we have further 419 performed correlational analyses between testosterone levels and striatal activities induced 420 by prosocial decisions for public vs. implicit baseline and for private vs. implicit baseline. 421 These analyses failed to reveal significant correlations between them (supplementary 422 Tables 1 and 2). Moreover, considering that our previous study has revealed that the 423 audience effect (public > private) for both prosocial choices and for selfish choices 424 commonly engaged a common brain network including the striatum (Qu et al., 2019), our 425 further correlation analysis revealed that similar results could also be observed for selfish 426 decisions for public > private condition (supplementary Figure 1). This provides further 427 evidence that such a relationship was not specific to prosocial decisions only but can also be 428 observed for selfish choices. Taken together, these results somewhat indicate that our 429 observation of a significant relationship between testosterone levels and audience-induced 430 striatal activities was not driven by the salience of the public context per se. Finally, to further 431 examine the potential relationship between testosterone levels and the difference in 
432 prosocial decisions made in public vs. in private for each type of organization, we performed

433 a number of correlational analyses. However, we did not observe any significant relationship 434 between them (POS ORG: $r=-0.07, p=0.78 ;$ NEG ORG: $r=0.18, p=0.44$ ) 435 (supplementary Figure 2). Similarly, when exploring the possible link between striatal 436 activities in public vs. in private and the difference in prosocial decisions made in public vs. 437 in private for each type of organization, we found a significant relationship between them 438 neither for the POS ORG (putamen: $r=0.02, p=0.94$; caudate: $r=-0.18, p=0.43$ ), nor for 439 the NEG ORG (putamen: $r=-0.16, p=0.50$; caudate: $r=0.03, p=0.90$ ). 


\section{Discussion}

The goal of the present study was to investigate the relationship between endogenous testosterone levels and the neural correlates responsible for prosocial decisions in presence of an audience, i.e., when social image and status concerns may be activated. Our results showed that striatal response correlated positively with endogenous testosterone levels in the public condition as compared to the private condition, regardless of organization types. That is, when being observed, a greater striatal activity correlated with testosterone levels. This effect highlights the fact that audience facilitates prosocial decisions for both types of organizations.

The striatum has been previously demonstrated to be one of the key brain areas involved in reputation-based behaviors, such as charitable giving and decision making in presence of a moral dilemma (Izuma, 2012; Izuma et al., 2010; Moll et al., 2006; Shenhav and Greene, 2010). This brain region is also strongly involved in reward processing (Haber and Knutson, 2009; Sescousse et al., 2013). To better understand the potential role of the striatum in public prosociality, two interdependent processes need to be considered during the process of reputation building. The first is the ability to create meta-representations of oneself so as to achieve the desirable image benefit from a given social behavior. A second process is the ability to overcome the conflict between the expected value of an option and the value of the other, less appealing, options (cost-benefit trade-off). While the right temporal parietal junction (TPJ) may contribute to each of these processes (Obeso et al., 2018), the striatum may preferentially be engaged in the cost-benefit analysis of the available options when image concerns are active (Izuma, 2012). This functional role of the striatum in reputation-based processes may be linked to the value attributed to rewards as a common denominator between prosocial behavior (monetary gains for the charity in the POS ORG) and moral behavior (moral benefit of rejecting offers in the NEG ORG). This was 
467 probably the case for both organizations when choices were made in public rather than in 468 private. In fact, Izuma et al. $(2008,2010)$ and Qu et al. (2019) have shown that making 469 donations while being observed and receiving monetary rewards both elicit striatal regions 470 activity. In addition, the striatum is known to be engaged upon recognition of acceptance 471 from others, i.e., being liked by others (Davey et al., 2010). The results of the current study 472 additionally reveal the neural mechanisms underlying the role of testosterone in public 473 prosociality when facing different moral dilemmas.

474 One important question is to identify the exact processes underlying the relationship 475 between testosterone levels and striatal activation. In our study, this process cannot be 476 attributed to the standard role of testosterone in reactive aggression. Yet, testosterone levels 477 have been shown to correspond with increased striatal activity related to monetary rewards 478 (Op de Macks et al., 2011). Because striatal activity is engaged with different types of 479 rewards (Li et al., 2015), including moral benefits, the observed correlation in the current 480 study could be proposed to reflect that testosterone potentiates striatal circuits functionality 481 to raise their reward functions, perhaps mediated through dopamine (Haber and Knutson, 482 2009). To sum up, our results could contribute to the understanding of the striatal functions 483 in contexts where social image is at play, and reveal a further striatal role in social 484 interactions, as testosterone levels might contribute to transform social image concerns into 485 generous or prosocial acts even for individuals that are not intrinsically prosocially 486 motivated.

487 Another possible contribution of the current findings to the literature relies on the 488 translation from women's to men's prosocial behavior in public. Previous studies have 489 shown the role of testosterone in status-enhancing behavior in women (Boksem et al., 2013; 490 Eisenegger et al., 2010; Mehta et al., 2015; van Honk et al., 2012; Zilioli et al., 2014). 491 However, it should be noted that these actual effects observed after testosterone treatment 
492 were induced by factors other than testosterone, since in the female brain aromatization to 493 estradiol could equally well mediate the behavioral effects. Also, testosterone administration 494 induces supra-physiological levels that are not representative for the actual natural level of 495 testosterone in the female brain. Our present study adds to the literature by showing how 496 natural testosterone is related to striatal activity during prosocial behavior induced by the 497 presence of an audience in men. This may suggest that the role of testosterone in social 498 behavior could be observed across sexes. However, several cognitive functions have been 499 proven different between men and women, as well as in temperament characteristics 500 (Borkenau et al., 2012a; Eagly, 2013). Men seem to show more of a variable pattern of 501 social characteristics than women such as in extraversion, or agreeableness levels, 502 suggesting that women have a less variable personality across the general population 503 (Borkenau et al., 2012b). These factors may induce sex differences in the interpretation of 504 social contexts. For example, sex differences were reported with regard to cortisol levels 505 disparity, which altered behavior differently in a competition context (Kivlighan et al., 2005).

506 As such, this raises an interesting question of whether our current findings in men would 507 extend to women.

508 Limitations

509 We acknowledge some limitations of our study. First, it included a relative small sample, 510 possibly tempering the strength of our conclusions. Replications with larger samples would 511 be welcome. Second, even though the present study had a strong prior hypothesis about the 512 striatum involved in the audience effect (Izuma et al., 2010; Moll et al., 2006), it will still be 513 useful to search for information about other brain regions since one region is unlikely to be 514 working all by itself. Third, although the measurement of total testosterone has been argued 515 to be effective in examining the relationship between testosterone and neuropsychological 516 function (Hua et al., 2016), further correlation with free testosterone would be needed to 
517 avoid limiting testing correlation to total testosterone levels, which may overlook the

518 possibility of excessive bondage to either sex-hormone binding globulin (SHBG) or albumin

519 in the blood. Fourth, we used blood samples to measure testosterone levels, which may 520 have activated anticipatory stress leading to increased cortisol levels. Moreover, there is a 521 great deal of interaction within the endocrine system, so our understanding of the 522 relationship between testosterone levels and the audience effect on prosocial behavior 523 would benefit from the inclusion of more hormones in the same study. In particular, the 524 dual-hormone hypothesis posits that testosterone's role in status-motivated behavior is 525 modulated by concentrations of cortisol (Dekkers et al., 2019; Mehta and Josephs, 2010; 526 Mehta and Prasad, 2015). Due to the small sample size, we were not able to explore 527 potential interacting effects of testosterone and cortisol on the audience effect on prosocial 528 behavior. Fifth, the present study only concerns men. We chose to scan only men because 529 gender has been shown to affect prosocial behavior (Buckholtz et al., 2015; Croson and 530 Gneezy, 2009; FeldmanHall et al., 2015) and unethical behavior (Berns et al., 2012; Dreber 531 and Johannesson, 2008; FeldmanHall et al., 2012). Moreover, young women experience 532 hormonal modulations of the reward system (Andreoni and Vesterlund, 2001; Dreher et al., 533 2007), which may affect the testosterone levels. In addition, there are known interactions 534 between the effects of audience and the observer's gender (kept constant in the present 535 experiment). For example, in women the mere presence of men can induce transient 536 decrements in cognitive efficiency and academic performances when confronted to math 537 tests despite similar performances when tested separately (Childs, 2012; Eckel and 538 Grossman, 1998). There is no doubt that future studies should investigate whether the 539 present findings extend to women. Sixth, although the present study provided novel insight 540 on the relationship between testosterone levels and audience effect on prosocial behavior 541 through striatal activity, it is only correlative evidence. Further investigations should explore 
542 the causal role of testosterone on the audience effect, using exogenous testosterone 543 administration.

544

545

546

547

548

549

550

551

552

553 554

\section{Conclusion}

The current study provides direct correlational neural evidence for prosocial image seeking in the striatum that is regulated by testosterone. These findings help with sheding light on prior findings showing that testosterone is involved in social status seeking in social endeavors. Our results constitute a good starting point for investigating the neural mechanisms underlying the causal role of testosterone in human social behaviors. Exploring the causal role of testosterone on the striatal activity induced by the audience effect by using exogenous testosterone administration would be a natural extension. 


\section{Acknowledgements}

Y. L. was supported by the National Natural Science Foundation of China (Grant Number 558 31600929), the Fundamental Research Funds for the Central Universities (Grant Number 559 010914380002) and a grant from Society for Social Psychology of Jiangsu Province (Grant 560 Number 20SSXGH006). This work has also benefited from the financial support of 561 IDEXLYON from Université de Lyon (project INDEPTH) within the Programme 562 Investissements d'Avenir (ANR-16-IDEX-0005) and of the LABEX CORTEX 563 (ANR-11-LABX-0042) of Université de Lyon, within the program Investissements d'Avenir 564 (ANR-11-IDEX-007) operated by the French National Research Agency. This work was 565 further supported by grant from the Fondation pour la Recherche Médicale FRM 566 DPA20140629796.

\section{Author contributions}

570 EM, MCV, LB and JCD contributed to the study concept and design. Testing and data 571 collection were performed by EM. EM, IO and YL performed the data analysis. YL drafted 572 the manuscript. IO, LB, MCV and JCD provided critical revisions of the manuscript for 573 submission. All authors approved the final version of the manuscript for submission.

575 Declarations of interest: none. 


\section{References}

Andreoni, J., Vesterlund, L., 2001. Which is the fair sex? Gender differences in altruism. The

580 Quarterly Journal of Economics 116, 293-312.

581 Archer, J., 2006. Testosterone and human aggression: an evaluation of the challenge 582 hypothesis. Neuroscience and biobehavioral reviews 30, 319-345.

583 Bateson, M., Nettle, D., Roberts, G., 2006. Cues of being watched enhance cooperation in a 584 real-world setting. Biol Lett 2, 412-414.

585 Beck, A.T., Beck, R.W., 1972. Screening depressed patients in family practice. A rapid 586 technic. Postgraduate Medicine 52, 81-85.

587 Berns, G.S., Bell, E., Capra, C.M., Prietula, M.J., Moore, S., Anderson, B., Ginges, J., Atran, 588 S., 2012. The price of your soul: neural evidence for the non-utilitarian representation of 589 sacred values. Philosophical Transactions of the Royal Society B: Biological Sciences 367, $590 \quad 754-762$.

591 Boksem, M.A.S., Mehta, P.H., Van den Bergh, B., van Son, V., Trautmann, S.T., Roelofs, K., 592 Smidts, A., Sanfey, A.G., 2013. Testosterone Inhibits Trust but Promotes Reciprocity. 593 Psychological Science 24, 2306-2314.

602 Bradley, A., Lawrence, C., Ferguson, E.J.P.o.t.R.S.B.B.S., 2018. Does observability affect 603 prosociality? 285, 20180116.

604 Buckholtz, J.W., Martin, J.W., Treadway, M.T., Jan, K., Zald, D.H., Jones, O., Marois, R., 605 2015. From blame to punishment: disrupting prefrontal cortex activity reveals norm 606 enforcement mechanisms. Neuron 87, 1369-1380.

607 Casto, K.V., Edwards, D.A., 2016. Testosterone and reconciliation among women: 608 after-competition testosterone predicts prosocial attitudes towards opponents. Adaptive 609 Human Behavior and Physiology 2, 220-233.

610 Chib, V.S., Adachi, R., O'doherty, J.P.J.S.c., neuroscience, a., 2018. Neural substrates of 611 social facilitation effects on incentive-based performance. 13, 391-403.

612 Childs, J., 2012. Gender differences in lying. Economics Letters 114, 147-149.

613 Coccaro, E.F., Beresford, B., Minar, P., Kaskow, J., Geracioti, T., 2007. CSF testosterone: 614 relationship to aggression, impulsivity, and venturesomeness in adult males with personality 615 disorder. Journal of psychiatric research 41, 488-492.

616 Croson, R., Gneezy, U., 2009. Gender differences in preferences. Journal of Economic 617 literature 47, 448-474.

618 Cutler, J., Campbell-Meiklejohn, D., 2019. A comparative fMRI meta-analysis of altruistic 619 and strategic decisions to give. Neurolmage 184, 227-241. 
620 Dabbs, J.M., Jr., Hargrove, M.F., 1997. Age, testosterone, and behavior among female 621 prison inmates. Psychosom Med 59, 477-480.

622 Davey, C.G., Allen, N.B., Harrison, B.J., Dwyer, D.B., Yücel, M., 2010. Being liked activates 623 primary reward and midline self - related brain regions. Human brain mapping 31, 660-668.

624 Déchaud, H., Lejeune, H., Garoscio-Cholet, M., Mallein, R., Pugeat, M., 1981. 625 Radioimmunoassay of testosterone not bound to sex-steroid-binding protein in plasma. 626 Clinical chemistry 35, 1609-1614.

627 Dekkers, T.J., van Rentergem, J.A.A., Meijer, B., Popma, A., Wagemaker, E., Huizenga, 628 H.M., 2019. A meta-analytical evaluation of the dual-hormone hypothesis: Does cortisol 629 moderate the relationship between testosterone and status, dominance, risk taking, 630 aggression, and psychopathy? Neuroscience \& Biobehavioral Reviews 96, 250-271.

631 Dreber, A., Johannesson, M., 2008. Gender differences in deception. Economics Letters 99, 632 197-199.

633 Dreher, J.-C., Dunne, S., Pazderska, A., Frodl, T., Nolan, J.J., O’Doherty, J.P., 2016. 634 Testosterone causes both prosocial and antisocial status-enhancing behaviors in human 635 males. Proceedings of the National Academy of Sciences 113, 11633.

636 Dreher, J.-C., Schmidt, P.J., Kohn, P., Furman, D., Rubinow, D., Berman, K.F., 2007. 637 Menstrual cycle phase modulates reward-related neural function in women. Proc Natl Acad 638 Sci U S A 104, 2465-2470.

639 Eagly, A.H., 2013. Sex differences in social behavior: A social-role interpretation. 640 Psychology Press.

641 Eckel, C.C., Grossman, P.J., 1998. Are women less selfish than men?: Evidence from 642 dictator experiments. The economic journal 108, 726-735.

643 Eisenegger, C., Haushofer, J., Fehr, E., 2011. The role of testosterone in social interaction. 644 Trends Cogn Sci 15, 263-271.

645 Eisenegger, C., Naef, M., Snozzi, R., Heinrichs, M., Fehr, E., 2010. Prejudice and truth 646 about the effect of testosterone on human bargaining behaviour. Nature 463, 356-359.

647 Everett, J.A.C., Faber, N.S., Crockett, M., 2015. Preferences and beliefs in ingroup 648 favoritism. Front Behav Neurosci 9, 15-15.

649 FeldmanHall, O., Dalgleish, T., Evans, D., Mobbs, D., 2015. Empathic concern drives costly 650 altruism. Neuroimage 105, 347-356.

651 FeldmanHall, O., Dalgleish, T., Thompson, R., Evans, D., Schweizer, S., Mobbs, D., 2012. 652 Differential neural circuitry and self-interest in real vs hypothetical moral decisions. Soc 653 Cogn Affect Neurosci 7, 743-751.

654 Geniole, S.N., Carré, J.M., 2018. Human social neuroendocrinology: Review of the rapid 655 effects of testosterone. Hormones and Behavior 104, 192-205.

656 Giammanco, M., Tabacchi, G., Giammanco, S., Di Majo, D., La Guardia, M., 2005. 657 Testosterone and aggressiveness. Medical science monitor : international medical journal of 658 experimental and clinical research 11, RA136-145.

659 Haber, S.N., Knutson, B., 2009. The reward circuit: linking primate anatomy and human 660 imaging. Neuropsychopharmacology 35, 4-26.

661 Hamilton, A.F.d.C., Lind, F., 2016. Audience effects: what can they tell us about social 662 neuroscience, theory of mind and autism? Culture and brain 4, 159-177. 
663 Hines, M., 2017. Gonadal Hormones and Sexual Differentiation of Human Brain and 664 Behavior.

665 Hua, J.T., Hildreth, K.L., Pelak, V.S., 2016. Effects of Testosterone Therapy on Cognitive 666 Function in Aging: A Systematic Review. Cogn Behav Neurol 29, 122-138.

667 Inoue, Y., Takahashi, T., Burriss, R.P., Arai, S., Hasegawa, T., Yamagishi, T., Kiyonari, T., 668 2017. Testosterone promotes either dominance or submissiveness in the Ultimatum Game 669 depending on players' social rank. Scientific Reports 7, 5335.

670 Izuma, K., 2012. The social neuroscience of reputation. Neuroscience research 72, 283-288.

671 Izuma, K., Matsumoto, K., Camerer, C.F., Adolphs, R., 2011. Insensitivity to social 672 reputation in autism. Proc Natl Acad Sci U S A 108, 17302-17307.

673 Izuma, K., Saito, D.N., Sadato, N., 2008. Processing of social and monetary rewards in the 674 human striatum. Neuron 58, 284-294.

675 Izuma, K., Saito, D.N., Sadato, N., 2010. Processing of the incentive for social approval in 676 the ventral striatum during charitable donation. J Cogn Neurosci 22, 621-631.

677 Kemp, A.H., Guastella, A.J., 2010. Oxytocin: prosocial behavior, social salience, or 678 approach-related behavior? Biological psychiatry 67, e33-e34.

679 Kivlighan, K.T., Granger, D.A., Booth, A., 2005. Gender differences in testosterone and 680 cortisol response to competition. Psychoneuroendocrinology 30, 58-71.

681 Li, Y., Ramoz, N., Derrington, E., Dreher, J.-C., 2020. Hormonal responses in gambling 682 versus alcohol abuse: A review of human studies. Progress in Neuro-Psychopharmacology 683 and Biological Psychiatry 100, 109880.

684 Li, Y., Sescousse, G., Amiez, C., Dreher, J.C., 2015. Local morphology predicts functional 685 organization of experienced value signals in the human orbitofrontal cortex. J Neurosci 35, 686 1648-1658.

687 Losecaat Vermeer, A.B., Krol, I., Gausterer, C., Wagner, B., Eisenegger, C., Lamm, C., 688 2020. Exogenous testosterone increases status-seeking motivation in men with unstable low 689 social status. Psychoneuroendocrinology 113, 104552.

690 Mazaika, P., Hoeft, F., Glover, G., Reiss, A.L., 2009. Methods and Software for fMRI 691 Analysis for Clinical Subjects. Human Brain Mapping.

692 Mazur, A., Booth, A., 1998. Testosterone and dominance in men. The Behavioral and brain 693 sciences 21, 353-363; discussion 363-397.

694 Mehta, P.H., Josephs, R.A., 2010. Testosterone and cortisol jointly regulate dominance: 695 Evidence for a dual-hormone hypothesis. Hormones and behavior 58, 898-906.

696 Mehta, P.H., Prasad, S., 2015. The dual-hormone hypothesis: a brief review and future 697 research agenda. Current Opinion in Behavioral Sciences 3, 163-168.

698 Mehta, P.H., van Son, V., Welker, K.M., Prasad, S., Sanfey, A.G., Smidts, A., Roelofs, 699 K.J.P., 2015. Exogenous testosterone in women enhances and inhibits competitive 700 decision-making depending on victory-defeat experience and trait dominance. 60, $701 \quad 224-236$.

702 Moll, J., Krueger, F., Zahn, R., Pardini, M., de Oliveira-Souza, R., Grafman, J., 2006. Human 703 fronto-mesolimbic networks guide decisions about charitable donation. Proceedings of the 704 National Academy of Sciences 103, 15623-15628. 
Mullard, A., 2009. What is the link between autism and testosterone? Nature.

Nadler, A., Camerer, C.F., Zava, D.T., Ortiz, T.L., Watson, N.V., Carré, J.M., Nave, G., 2019. Does testosterone impair men's cognitive empathy? Evidence from two large-scale randomized controlled trials. Proceedings of the Royal Society B: Biological Sciences 286, 20191062.

Nave, G., Nadler, A., Dubois, D., Zava, D., Camerer, C., Plassmann, H., 2018. Single-dose testosterone administration increases men's preference for status goods. Nat Commun 9, 2433.

Newman, M.L., Sellers, J.G., Josephs, R.A., 2005. Testosterone, cognition, and social status. Hormones and behavior 47, 205-211.

Obeso, I., Moisa, M., Ruff, C.C., Dreher, J.-C.J.e., 2018. A causal role for right temporo-parietal junction in signaling moral conflict. 7, e40671.

Oldfield, R.C., 1971. The assessment and analysis of handedness: the Edinburgh inventory. Neuropsychologia 9, 97-113.

Op de Macks, Z.A., Moor, B.G., Overgaauw, S., Güroğlu, B., Dahl, R.E., Crone, E.A., 2011. Testosterone levels correspond with increased ventral striatum activation in response to monetary rewards in adolescents. Developmental Cognitive Neuroscience 1, 506-516.

Qu, C., Météreau, E., Butera, L., Villeval, M.C., Dreher, J.-C., 2019. Neurocomputational mechanisms at play when weighing concerns for extrinsic rewards, moral values, and social image. PLOS Biology 17, e3000283.

Räsänen, P., Hakko, H., Visuri, S., Paanila, J., Kapanen, P., Suomela, T., Tiihonen, J., 1999. Serum testosterone levels, mental disorders and criminal behaviour. Acta psychiatrica scandinavica 99, 348-352.

Renfree, M.B., Wilson, J.D., Shaw, G., 2002. The hormonal control of sexual development, Novartis Foundation Symposium. Chichester; New York; John Wiley; 1999, pp. 136-156.

Rinaldi, S., Dechaud, H., Biessy, C., Morin-Raverot, V., Toniolo, P., Zeleniuch-Jacquotte, A., Akhmedkhanov, A., Shore, R.E., Secreto, G., Ciampi, A., Riboli, E., Kaaks, R., 2001. Reliability and validity of commercially available, direct radioimmunoassays for measurement of blood androgens and estrogens in postmenopausal women. Cancer epidemiology, biomarkers \& prevention : a publication of the American Association for Cancer Research, cosponsored by the American Society of Preventive Oncology 10, 757-765.

Rowe, R., Maughan, B., Worthman, C.M., Costello, E.J., Angold, A., 2004. Testosterone, antisocial behavior, and social dominance in boys: pubertal development and biosocial interaction. Biological psychiatry 55, 546-552.

Sabot, J.F., Deruaz, D., Dechaud, H., Bernard, P., Pinatel, H., 1985. Determination of plasma testosterone by mass fragmentography using [3,4-13C]testosterone as an internal standard. Journal of Chromatography 339, 233-242.

Sanchez-Pages, S., Turiegano, E., 2010. Testosterone, facial symmetry and cooperation in the prisoners' dilemma. Physiology \& behavior 99, 355-361.

Sekiguchi, Y., Hata, T.J.B.P., 2018. Effects of the mere presence of conspecifics on the motor performance of rats: Higher speed and lower accuracy.

Sellers, J.G., 2006. Testosterone and status seeking. 
748 Sescousse, G., Caldú, X., Segura, B., Dreher, J.-C., 2013. Processing of primary and 749 secondary rewards: A quantitative meta-analysis and review of human functional 750 neuroimaging studies. Neuroscience \& Biobehavioral Reviews.

751 Sescousse, G., Li, Y., Dreher, J.-C., 2015. A common currency for the computation of 752 motivational values in the human striatum. Soc Cogn Affect Neurosci 10, 467-473.

753 Shenhav, A., Greene, J.D., 2010. Moral judgments recruit domain-general valuation 754 mechanisms to integrate representations of probability and magnitude. Neuron 67, 667-677.

755 van Honk, J., Bos, P.A., Terburg, D., 2014. Testosterone and dominance in humans: 756 behavioral and brain mechanisms, New frontiers in social neuroscience. Springer, pp. 757 201-214.

758 van Honk, J., Montoya, E.R., Bos, P.A., van Vugt, M., Terburg, D., 2012. New evidence on 759 testosterone and cooperation. Nature 485, E4.

760 van Honk, J., Will, G.-J., Terburg, D., Raub, W., Eisenegger, C., Buskens, V., 2016. Effects 761 of Testosterone Administration on Strategic Gambling in Poker Play. Scientific Reports 6, 76218096.

763 Zethraeus, N., Kocoska-Maras, L., Ellingsen, T., Von Schoultz, B., Hirschberg, A.L., 764 Johannesson, M., 2009. A randomized trial of the effect of estrogen and testosterone on 765 economic behavior. Proceedings of the National Academy of Sciences 106, 6535-6538.

766 Zilioli, S., Mehta, P.H., Watson, N.V., 2014. Losing the battle but winning the war: uncertain 767 outcomes reverse the usual effect of winning on testosterone. Biol Psychol 103, 54-62. 
771 Figure 1. Experimental design. We used a $2 \times 2$ within-participant design, in which 772 participants decide to accept or reject the possibility of doing a costly good action for the 773 benefit of a positively evaluated organization (POS ORG) or avoiding a bad one which would 774 advantage both them and negatively evaluated organization (NEG ORG), either in presence 775 or absence of an audience (PUBLIC vs. PRIVATE). The amounts of the potential transfers to 776 the organizations and of the potential costs or payoffs to the participants were varied 777 independently across trials. In each trial, the organization potential gains ranged from 4 to 32 778 Euros, by steps of 4 Euros. The participants' potential payoffs (in the case of the NEG ORG) 779 or costs (in the case of the POS ORG) varied from 1 to 8 Euros, by steps of 1 Euro. This 780 manipulation resulted in 64 different dilemmas. Each trial began with the presentation of an 781 offer that the participant could either accept or reject by pressing the left button response or 782 the right button response, respectively. To further stress the presence of observers during 783 public trials, the chosen alternative was highlighted for $1.5 \mathrm{~s}$ by expanding its characters, 784 while the other was disappearing. On the opposite, in the private condition, no changes were 785 shown after the response, ensuring the participant that nobody would be able to see their 786 choice. A fixation cross was eventually displayed during a random time interval.

787 Figure 2. Behavioral results. (A) Decisions modulated by the presence of an audience. 788 The participants' rate of acceptance was significantly increased when decisions were 789 observed in public than in private for the POS ORG. Similarly, for the NEG ORG, 790 participants were significantly more likely to reject the propositions in public than in private.

791 The results indicated that participants made status seeking behavior due to the presence of 792 an observer. POS ORG, positively evaluated organization; NEG ORG, negatively evaluated 793 organization. ${ }^{*} p<0.01,{ }^{\star} p<0.05$. Error bars represent standard errors of the mean. (B) 794 Color-coded heatmaps of the probability of acceptance to donate for each dilemma of 
795 the $\mathbf{8 x} \mathbf{8}$ monetary/moral gain/loss matrix. Warmer colors indicate higher probability of 796 acceptance, whereas colder colors indicate lower probability of acceptance. One heatmap is 797 drawn for each type of organization and each audience condition.

798 Figure 3. The correlation between testosterone levels and striatal activity. Activation in 799 the striatum (putamen: $\mathrm{MNI}[\mathrm{x}$ y z $][-215-11], \mathrm{T}=6.77, p(\mathrm{SVC})<0.05$, FWE; caudate nucleus:

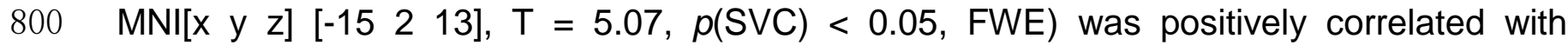
801 testosterone levels, regardless of the types of organization. The scatter plots indicate that 802 the striatum involved in decisions about transferring to the POS ORG and NEG ORG 803 respectively in public is particularly prominent in high-testosterone men. POS ORG, 804 positively evaluated organization; NEG ORG, negatively evaluated organization. 


\section{Table Legends}

806 Table 1. Foci of activation relating to decisions made in public as compared to that made in 807 private and vice versa. All reported foci are thresholded at $p<0.001$ voxel-wise uncorrected 808 with $p<0.05$ FWE cluster-wise correction except for regions marked with the sign * which 809 survived at a SVC corrected threshold of $p<0.05$, FWE.

810 Table 2. Foci of activation relating to the correlation between brain activity induced by 811 decisions made in public vs that made in private and the testosterone levels for both 812 organizations. All reported foci are thresholded at $p<0.001$ voxel-wise uncorrected with $p<$

8130.05 FWE cluster-wise correction except for regions marked with the sign * which survived at 814 a SVC corrected threshold of $p<0.05$, FWE. 


\section{Supplemental materials:}

817

818

819 Supplementary Table 1. Foci of activation relating to the correlation between brain activity

820 induced by prosocial decisions for public vs. implicit baseline and the basal testosterone

821 levels for both organizations. All reported foci are thresholded at $p<0.001$ cluster-wise 822 uncorrected $(\mathrm{k}>10)$.

823

\begin{tabular}{|c|c|c|c|c|c|}
\hline \multirow{3}{*}{ Brain regions } & \multirow{3}{*}{ L/R } & \multicolumn{3}{|c|}{ MNI coordinates } & \multirow{3}{*}{ T value } \\
\hline & & & & & \\
\hline & & $\mathbf{x}$ & $y$ & Z & \\
\hline \multicolumn{6}{|c|}{ Prosocial decisions: (public > implicit baseline) x testosterone levels } \\
\hline Middle temporal gyrus & $\mathrm{R}$ & 51 & -1 & -23 & 5.12 \\
\hline Superior parietal gyrus & L & -15 & -34 & 37 & 4.63 \\
\hline Posterior cingulate gyrus & L & -34 & 43 & 26 & 4.31 \\
\hline Superior parietal gyrus & $\mathrm{R}$ & 15 & -40 & 58 & 4.43 \\
\hline \multicolumn{6}{|c|}{ Prosocial decisions: (implicit basline $>$ public) x testosterone levels } \\
\hline Lingual gyrus & L & -97 & -5 & 64 & 4.81 \\
\hline
\end{tabular}


827 Supplementary Table 2. Foci of activation relating to the correlation between brain activity 828 induced by prosocial decisions for private vs. implicit baseline and the basal testosterone 829 levels for both organizations. All reported foci are thresholded at $p<0.001$ cluster-wise 830 uncorrected $(k>10)$.

831

\begin{tabular}{|c|c|c|c|c|c|}
\hline \multirow{3}{*}{ Brain regions } & \multirow{3}{*}{ L/R } & \multicolumn{3}{|c|}{ MNI coordinates } & \multirow{3}{*}{ T value } \\
\hline & & & & & \\
\hline & & $\mathbf{X}$ & $y$ & z & \\
\hline \multicolumn{6}{|c|}{ Prosocial decisions: (private > implicit baseline) x testosterone levels } \\
\hline Postcentral gyrus & $\mathrm{R}$ & 51 & -10 & 52 & 5.14 \\
\hline Anterior insula & $\mathrm{R}$ & 33 & -1 & -8 & 5.03 \\
\hline Middle frontal gyrus & L & -42 & 14 & 52 & 4.98 \\
\hline \multicolumn{6}{|c|}{ Prosocial decisions: (implicit basline > private) $x$ testosterone levels } \\
\hline Lingual gyrus & L & -3 & -82 & -14 & 5.66 \\
\hline Cerebellum & L & -12 & -37 & -26 & 5.19 \\
\hline Midbrain & $\mathrm{R}$ & 12 & -19 & -14 & 4.98 \\
\hline
\end{tabular}



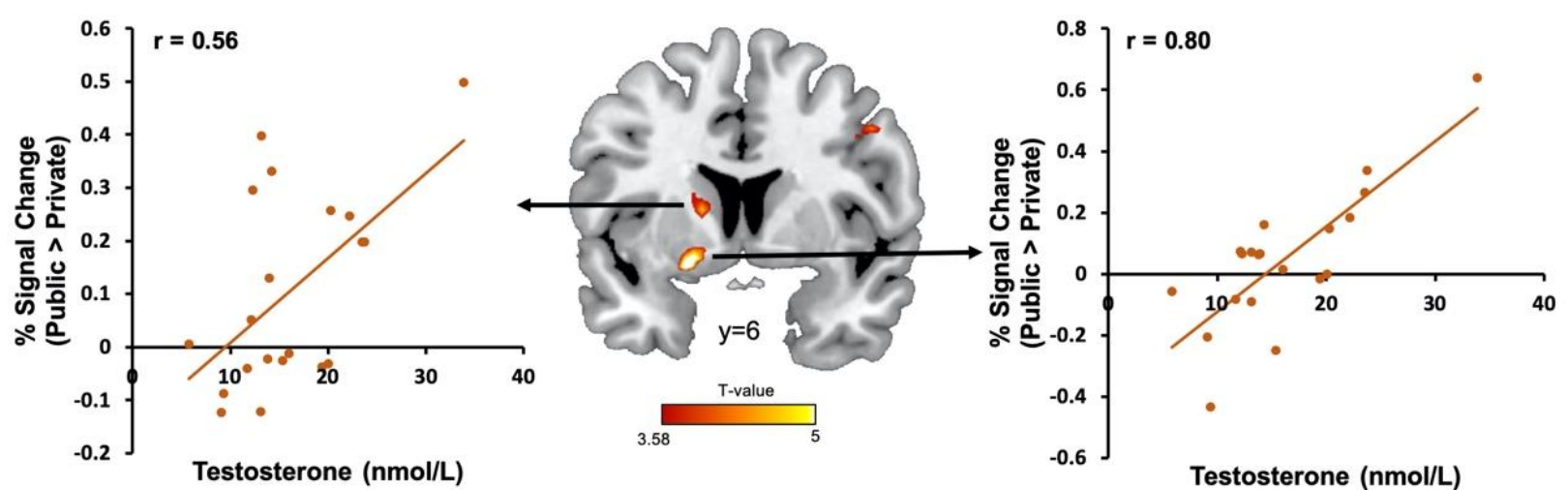

Supplementary Figure 1. The scatter plot showing the relationship between testosterone levels and striatal activity induced by selfish decisions made in public >

843 private condition. Activation in the striatum (putamen: MNI[x y z] [-21 5 -11], T=6.77, $844 p(\mathrm{SVC})<0.05$, FWE; caudate nucleus: MNI[x y z] [-15 2 13], T = 5.07, $p(\mathrm{SVC})<0.05, \mathrm{FWE})$ 845 was positively correlated with testosterone levels, regardless of the types of organization. 846 The scatter plots indicate that the striatum involved in selfish decisions about transferring to 847 the POS ORG and NEG ORG respectively in public > in private is particularly prominent in 848 high-testosterone men. 